\title{
Das Narrativ der dissoziativen Identitätsstörung im Kontext ökonomischer Imperative
}

»Identität ist immer weniger monolithisch, sondern nur noch plural möglich. Leben unter heutigen Bedingungen ist Leben im Plural, will sagen: Leben im Übergang zwischen unterschiedlichen Lebensformen. «1

Hybridität, Heterogenität, Pluralisierung, Diversität, Fragmentierung und das Multiple sind Schlagworte gegenwärtiger Identitätsdiskurse, derer sich die ProtagonistInnen neoliberaler Transformationsprozesse gleichermaßen bedienen wie die VertreterInnen postkolonialer Herrschaftskritik, queer-feministischer Positionen, soziologischer Ansätze oder der Theoreme der Kognitionswissenschaften. In derlei Konzepten gerät das Multiple zu einem weitgehend affirmierten Modell, welches gerade der (postmodernen) condition humaine gerecht werden soll. Dabei wird aus soziologischer Sicht oftmals kritisch argumentiert, dass die veränderten gesellschaftlichen Verhältnisse der spätmodernen Multioptionsgesellschaft ${ }^{2}$ respektive des Spätkapitalismus ${ }^{3}$, des Zeitalters elektronischer Massenmedien und virtueller Welten ${ }^{4}$ die Multiplizität des Menschen notwendig machen oder zumindest fördern.

Parallel zu dieser gehäuften Ortung des Multiplen steigt auch die Zahl der psychiatrischen Diagnosen der sogenannten dissoziativen Identitätsstörung (vormals multiple Persönlichkeit) seit Mitte der 1980er Jahre vornehmlich in den USA in einer Weise an, dass die Wochenzeitung Die Zeit Ende der 1990er Jahre polemisch von einer »amerikanischen Markenkrankheit « beziehungsweise einem »veritablen Massenwahn « berichten konnte. Wurde bis 1980 weltweit lediglich über etwa 200 Fälle multipler Persönlichkeit in der psychiatrischen Literatur berichtet, so beläuft sich die Zahl der von 1980 an dokumentierten Fälle mittlerweile bereits auf mehrere Zehntausend. $^{5}$

Im Folgenden soll anhand der Zusammenschau der postmodernen Position des Philosophen Wolfgang Welsch, Ulrich Bröcklings Konzept des unternehmerischen Selbst sowie Richard Sennetts Entwurf des flexiblen Menschen aufgezeigt werden, dass gerade das psychiatrische Narrativ der multiplen Persönlichkeit als paradigmatisches Leitbild einer Identität im Übergang - eines, so Welsch, neuen »Typus

1 Welsch 1991, S. 171.

2 Gross 1994; Gergen 1991.

3 Sennett 1998; Bauman 1997.

4 Turkle 1995.

5 Vgl. Hacking 1995.

Leviathan, 42. Jg., 2/2014, S. $191 \div 218$ 
von Subjektivität « ${ }^{6}$ - dienen kann, das der steigenden Pluralität sowie Pluralitätsanforderung der Welt gerecht zu werden verspricht. Zugleich, und hier dienen Bröcklings und Sennetts Ansätze als eine Art Brücke zwischen Welschs philosophischer Position und dem gegenwärtigen (psychiatrischen) Multiplendiskurs, lässt sich seit den späten 1980er Jahren im Rahmen einer umfassenden Ökonomisierung des Sozialen auch eine Ökonomisierung alternativer Subjektformen feststellen. ${ }^{7}$ Diese Dynamik der Ökonomisierung des vormals Devianten, so die grundlegende These des vorliegenden Beitrags, betrifft im hohen Maße das Narrativ der multiplen Persönlichkeit, und es lässt sich folglich fragen, ob dessen Popularität nicht gerade darauf basiert, dass das dissoziative Subjekt als eine mögliche zeitgerechte Form des Homo oeconomicus betrachtet werden kann. Im Zuge dieser Fragestellungen gilt es der Annahme nachzugehen, dass gerade dasjenige, was den Störungszustand dissoziativer Identität zuvorderst als pathologisch kennzeichnet, sich zugleich - aufgrund ökonomischer Vorteile - in hohem Maße dazu eignet, zu affirmierten Charakteristika eines neuen Leistungstyps umgewertet zu werden. Die multiple Persönlichkeit korreliere demnach genuin mit neoliberalen Leistungsimperativen wie etwa jenen der individuellen Flexibilität, des Werterelativismus und der »kreativen Selbstgestaltung ". Die Ambivalenz dieser »Modekrankheit " zwischen Verwerfung, die einerseits exemplarisch in der postulierten Notwendigkeit einer (Re-)Integration und andererseits in der nach wie vor präsenten $\mathrm{Kritik}^{8}$ an der Diagnose zum Ausdruck kommt, und deren Affirmation, wie sie in zahlreichen Autobiografien von Multiplen, Blogs 9 und Selbsthilfegruppen ${ }^{10}$ insbesondere seit den 1980er Jahren zutage tritt, mag gerade in diesem Versprechen der Leistungssteigerung eine wesentliche Ursache haben.

6 Welsch 1993, S. 171.

7 Exemplarisch sei hier die Ökonomisierung von Autismus genannt, der spätestens seit dem Film Rain Man (1988) sowohl in Fachdiskursen als auch in der Populärkultur in den Kontext von sogenannten Inselbegabungen und Genialität gestellt wird.

8 So ist die Fachwelt gewissermaßen selbst auffallend gespalten, da es auf der einen Seite vehemente VerfechterInnen der Diagnose gibt (z.B. Putnam 2013 [1989]; Ross 1989; Herman 1992), auf der anderen Seite hält sich auch in psychiatrischen Kreisen hartnäckig die These, die dissoziative Identitätsstörung sei iatrogen, das heißt von TherapeutInnen suggeriert, und damit eher sozial beziehungsweise kulturell erzeugt denn eine reale psychische Störung (z.B. Aldridge-Morris 1991; Dale 1999; Held 1999).

9 Vgl. beispielsweise die Blogs Living successfully with Dissociative Identity Disorder. A daily/weekly blog about successfully living with Dissociative Identity Disorder and the daily challenges of living with this condition. http://suzy-livingsucessfullywithdid.blogspot.co.at/ (Zugriff vom 31.03.2014), Life with Dissociative Identity Disorder stories and struggles from my life with DID. http://lifewithdissociativeidentitydisorder.blogspot.co.at/ (Zugriff vom 31.03.2014) oder Liz Elliot's blog. A positive life with Dissociative Identity Disorder (D.I.D.). http://lizelliot.wordpress.com/ (Zugriff vom 31.03.2014).

$10 \mathrm{Vgl}$. hierzu beispielsweise Astraea's web. Multiple personality resources \& controversies. www.astraeasweb.net/plural/ (Zugriff vom 31.03.2014). 
Im zweiten Teil des Textes gilt es die genannten Thesen anhand ausgewählter Autobiografien, der Textsorte der psychiatrischen Multiplenbewegung seit den 1980er Jahren, darzulegen. Zugleich sollen die erwähnten Leistungsimperative präzisiert und mit Hilfe von Beispielen veranschaulicht werden.

\section{Dissoziative Identitätsstörung}

»Vermutlich habe ich noch nie einen Patienten gehabt, der einerseits so gut funktioniert und andererseits so bedürftig ist. ${ }^{11}$

Die dissoziative Identitätsstörung kann auf zwei Hochphasen zurückblicken, während sie in der Zeit dazwischen weitgehend in Vergessenheit gerät: ihre Entstehungszeit und erste Blüte von 187512-1910, wo sie als dédoublement de la personalité, alternierende Persönlichkeit oder double consciousness den zentralen Bestandteil des psychiatrischen Denkens darstellte, sowie ihre Renaissance in den 1970er Jahren, die bis in die Gegenwart anhält. Während sie im 19. beziehungsweise beginnenden 20. Jahrhundert von Theoretikern wie insbesondere Alfred Binet und Pierre Janet in Frankreich, Morton Prince in den USA oder auch dem frühen Siegmund Freud weitgehend der Hysterie zugerechnet wurde, ${ }^{13}$ deren "Niedergang " ihr dadurch mit beschieden war, ${ }^{14}$ bildete sich in den 1970er Jahren aufgrund zunächst noch vereinzelter Fälle erneut eine psychiatrische "Multiplenbewegung ", welche die multiple Persönlichkeitsstörung nun als eigenständiges Störungsbild auszumachen suchte. ${ }^{15} 1980$ wurde die multiple Persönlichkeitsstörung in das amerikanische Diagnosehandbuch für Geisteskrankheiten (DSM III) und 1992 in die von der WHO

11 Casey, Wilson 1995 [1991], S. 98.

12 Die erste systematische Beschreibung einer Doppelpersönlichkeit, die der Französin Félida X., erfolgte 1875 durch den Mediziner Eugène Adam.

13 Vgl. Ellenberger 1970.

14 Vgl. Hacking 1995.

15 Die gängige Narration hierzu innerhalb des psychiatrischen Diskurses lautet wie folgt: »Durch das Engagement und die harte Arbeit einer kleinen Gruppe von Klinikern, die ihr Ziel zunächst isoliert und unabhängig voneinander verfolgten, jedoch später zunehmend kooperierten und einander unterstützten, gelang es, die DIS wieder in den Rang einer anerkannten klinischen [sic!] Störung zu erheben "(Putnam 2013 [1989], S. 54). Als die wichtigsten theoretischen Anstöße nennt Putnam Ellenbergers Studie The discovery of the unconscious. The history and evolution of dynamic psychiatry (Ellenberger 1970), die sich sehr ausführlich mit dem Phänomen des doppelten Bewusstseins im 19. Jahrhundert und dessen zentraler Einflussnahme auf moderne Auffassungen des Psychischen beschäftigt, sowie den von der amerikanischen Psychoanalytikerin Cornelia Wilbur dokumentierten und von Flora Rheta Schreiber in Buchform verfassten Fall Sybil (Schreiber 2006 [1974]). Auf dieses von ihm als »klinische Pflichtlektüre « bezeichnete Buch Bezug nehmend, stellt Putnam fest: »Das Buch Sybil [...] wurde zu einer Art Modell, mit dem man andere Patienten vergleichen konnte, um sie besser zu verstehen « (Putnam 2013 [1989], S. 55).

Leviathan, 42. Jg., 2/2014 
publizierte Internationale Klassifikation von Krankheiten (ICD-10) aufgenommen und floss damit international in die Curricula der Psychiatrieausbildung ein. 1994 wurde das Störungsbild in der vierten Auflage des amerikanischen Handbuchs (DSM IV) in dissoziative Identitätsstörung umbenannt, darüber hinaus wurden einige Veränderungen in der phänomenologischen Festlegung desselben vorgenommen. Das DSM IV führt folgende vier Kriterien zur Diagnostizierung der Störung (F44.81) an:
»A. Die Anwesenheit von zwei oder mehr unterscheidbaren Identitäten oder Persönlich- keitszuständen (jeweils mit einem eigenen, relativ überdauernden Muster der Wahrnehmung von, der Beziehung zur und dem Denken über die Umgebung und das Selbst).
B. Mindestens zwei dieser Identitäten oder Persönlichkeitszustände übernehmen wiederholt die Kontrolle über das Verhalten der Person.
C. Eine Unfähigkeit, sich an wichtige persönliche Informationen zu erinnern, die zu umfas- send ist, um durch gewöhnliche Vergeßlichkeit erklärt zu werden.
D. Die Störung geht nicht auf die direkte körperliche Wirkung einer Substanz (z. B. Black- outs oder ungeordnetes Verhalten während einer Alkoholintoxikation) oder eines medizi- nischen Krankheitsfaktors zurück (z. B. komplex-partielle Anfälle).«16

Zentrale TheoretikerInnen der zeitgenössischen "Multiplenbewegung " wie Richard Kluft, Frank Putnam oder Colin Ross beziehen sich auch heute noch weitgehend auf Pierre Janet, soweit es die theoretische Einordnung betrifft. Mit dieser historischen Verankerung geht zum einen eine Abkehr von der Freud'schen Psychoanalyse und deren triebtheoretischem Denkmodell einher, zum anderen dient der Rekurs auf Janets Konzept der dissociation der Abgrenzung von Schizophrenie, welche, so die häufige Lesart innerhalb des gegenwärtigen Multiplendiskurses, die Erforschung und Diagnose des doppelten Bewusstseins historisch verdrängt habe ${ }^{17}$ und bis heute, gemeinsam mit dem Borderline-Syndrom, zu häufigen Fehldiagnosen führe. Anders jedoch als die Schizophrenie wird die dissoziative Identitätsstörung als Traumafolgestörung verstanden, der meist schwerer (sexueller) Missbrauch in der frühen Kindheit, das heißt zu einer Zeit, wo sich die Persönlichkeit noch nicht voll entwickeln konnte, zugrunde liegt. ${ }^{18}$ Aus Ermangelung einer Alternative, sich solchen anhaltenden existenziell bedrohlichen Situationen zu entziehen, beginne das Kind damit, Persönlichkeitsanteile, Erinnerungen, Erlebnisse etc. von sich amnestisch abzuspalten, um auf diese Weise ein Überleben als Person zu ermöglichen. Dissoziation wird folglich in der Forschungsliteratur weniger als Störung denn als kreative Schutzfunktion begriffen, die prinzipiell jedem Menschen, allerdings in unterschiedlichem Ausmaß, zugänglich ist. Dementsprechend lautet auch die Erklärung in einer der im Anschluss behandelten Autobiografien: »Eine

16 Saß et al. 1998, S. 210.

17 Vgl. die von Rosenbaum (1980) angestoßene Debatte zu dem historischen Verhältnis von Schizophrenie und multipler Persönlichkeit.

18 Hacking sieht in dieser kausalen Zusammenführung von Missbrauch und multipler Persönlichkeit eine wesentliche Ursache für die exponentielle Zunahme der Diagnoserate seit den 1980er Jahren, indem er letztere mit der aus den USA kommenden Antimissbrauchsbewegung in Verbindung setzt. Dahingehend stellt er fest: »Nur eine Gesellschaft, die anzuerkennen bereit ist, daß familiäre Gewalt überall vorkommt, konnte überall multiple Persönlichkeiten finden« (Hacking 1995, S. 16). 
multiple Persönlichkeit zu entwickeln, ist die letzte Überlebensstrategie intelligenter Kinder, deren Erinnerungen unerträglich sind $« .{ }^{19}$ Die abgespaltenen Anteile, sogenannte Alters, entwickeln sich je nach Komplexität und Quantität der jeweiligen Außenzeit, das heißt der Kontrolle über den Körper, zu eigenständigen »Alter-Persönlichkeiten « beziehungsweise zu bloßen Persönlichkeitsfragmenten. Diese verfügen in der Regel über individuelle Erinnerungen, soziale Beziehungen und Verhaltensmuster in unterschiedlicher Komplexität. Dabei variiert die Anzahl der Alters von zwei bis zu mehreren hundert, die sich der Existenz einiger oder aller anderen Persönlichkeiten in unterschiedlichem Ausmaß bewusst sein können beziehungsweise mit diesen in Kontakt treten können. Während das Ziel der meisten Therapien die Fusion ${ }^{20}$ darstellt, lässt sich innerhalb des Multiplendiskurses, insbesondere im Rahmen der zahlreichen Autobiografien, auch das Gegenmodell einer »funktionalen Multiplizität " ausmachen, das heißt eines Zustands, in dem die amnestischen Barrieren zwischen den verschiedenen Alter-Persönlichkeiten mittels Therapie weitgehend überwunden werden konnten, wodurch ein kollektiv steuerbares System der Vielheit entsteht. Diese Form der Multiplizität wird von Betroffenen häufig auch als »Healthy Multiplicity « verstanden und auf diese Weise von der Zuschreibung einer Störung abgegrenzt. ${ }^{21}$ In diesem Zustand wird Multiplizität oft von Betroffenen selbst, wie im letzten Abschnitt anhand zweier Autobiografien exemplarisch aufzuzeigen sein wird, als Mehrwert empfunden, den es im Sinne eines alternativen Wesensmodells zu erhalten gilt. Dieser Mehrwert, so eine zentrale These des vorliegenden Textes, ist in erster Linie ökonomischer Natur und definiert sich über Schlagworte wie Flexibilität, Effizienzsteigerung und Arbeitskraftzuwachs. Für den vorliegenden Artikel ist dementsprechend insbesondere eine solche Auffassung von »funktionaler Multiplizität « von Interesse. Dieser Fokus soll nicht über die zentrale Rolle von Traumata und das konkrete Leiden als »multiple Persönlichkeit « hinwegtäuschen, aber diese Aspekte werden bei der gewählten Fragestellung nicht im Vordergrund stehen. An dieser Stelle ist grundsätzlich festzustellen, dass mit der Schwerpunktsetzung auf die semi-fiktionale Gattung der Autobiografie gezielt das Narrativ der multiplen Persönlichkeit in das Blickfeld genommen wird und nicht empirisch beobachtbare Subjekte in psycho-sozialen Einrichtungen. Es geht folglich nicht darum, inwiefern dieser »Idealtypus « dissoziativer Identität der lebensweltlichen Realität tatsächlich Betroffener entspricht, sondern vielmehr, welche ökono-

\section{Oxnam 2008 [2005], S. 61.}

20 In den 1980er Jahren wurde innerhalb des psychiatrisch-therapeutischen Diskurses die Integration beziehungsweise Fusion nicht zwingend als Therapieziel verfolgt, was mitunter darauf zurückzuführen ist, dass den Alter-Persönlichkeiten eine stärkere Eigenständigkeit und Komplexität zugesprochen wurde. Seit den 1990er Jahren besteht jedoch die gängige Lehrmeinung, dass es sich bei den abgespaltenen Persönlichkeiten lediglich um Fragmente handle, wodurch nicht mehr, sondern letztlich weniger als eine Person vorhanden sei. Die Integration führe in diesem Sinne erst zur Herausbildung einer vollständigen Person. Diese Entwicklung innerhalb des Psychiatriediskurses ging mit der Umbenennung des Erscheinungsbilds in dissoziative Identitätsstörung einher.

21 Vgl. beispielsweise http://healthymultiplicity.com/ (Zugriff vom 31.03.2014).

Leviathan, 42. Jg., 2/2014 
mischen Implikationen der »Erzählung « der multiplen Persönlichkeit diskursiv eingeschrieben sind. ${ }^{22}$

\section{Multiplizität als Imperativ der Gegenwart}

»Pluralitätskompetenz wird zu einer Bedingung gelingender Subjektivität. «23

In den Aufsätzen »Subjektsein heute ${ }^{24}$ und »Identität im Übergang « 25 diagnostiziert der deutsche Philosoph Wolfgang Welsch für die Gegenwart ein »Leben im Plural ${ }^{26}$, das nicht nur soziologisch als ein »inmitten « multipler sozialer und kultureller Kontexte gefasst wird, sondern das »in sich " strukturelle Vielheit birgt. Die Ursache sieht Welsch zum einen in der pragmatischen Notwendigkeit, um in »einer plural verfassten Sozietät « überhaupt » handlungsfähig « ${ }^{27}$ zu bleiben. Zum anderen erklärt er die »Pluralisierung des Subjekts « auch insofern als normativ geboten, um die Pluralität der Welt respektive der Gesellschaft gemäß einer demokratischen Verfassung entsprechend anerkennen und verteidigen zu können. Zuletzt verweist er auf die Relevanz einer internen Pluralität für die freie Selbstgestaltung von Individuen - wenn »man entdeckt - mit einem Gefühl der Überraschung und Befreiung - , dass man auch anders sein könnte «. ${ }^{28}$

Als Sinnbilder dieser »Identität im Übergang « beziehungsweise solcher »Identitätsvervielfachungen ${ }^{29}$ dienen dem Autor gerade psychiatrische Formen des Identitätsverlusts, die, weitgehend von ihrer Devianz befreit, nun zum »Verstehensschlüssel für Normalität « ${ }^{30}$, zur » Matrix heutiger Individuen $\aleph^{31}$ geworden sind. So Welsch: »Man wird zunehmend versuchen müssen, die Kranken ihre Divergenz als normalitätsnah erfahren zu lassen «.32 Dementsprechend propagiert er nicht eine Normalisierung dieser Divergenz, sondern eine »Versöhnung des Kranken mit sei-

22 Es wird im Folgenden die Frage nach dem Realitätsgehalt der untersuchten Quellen, das heißt sowohl nach deren »Authentizität " als auch nach deren Glaubwürdigkeit, nicht untersucht, und es wird auch nicht der Frage nachgegangen, ob die dissoziative Identitätsstörung iatrogen verursacht ist oder nicht.

23 Welsch 1993, S. 159.

24 Welsch 1993.

25 Welsch 1991.

26 Welsch 1993, S. 159.

27 Ebd., S. 162.

28 Ebd. Welschs Argumentation weist in dem Nebeneinander aus Notwendigkeit und Freiheit einen Widerspruch auf, der sich innerhalb des Textes nicht auflöst.

29 Welsch 1991, S. 172.

30 Ebd., S. 171.

31 Ebd., S. 199.

32 Ebd. 
nem Widerspruch «, eine »Bestärkung seiner divergierenden Selbstseinsfähigkeiten $\ll .33$

Widmet man sich Welschs Texten eingehender, so zeigt sich, dass insbesondere die dissoziative Identitätsstörung, wenn auch nicht explizit von ihm benannt, als modellhaft für sein Konzept pluraler Identität betrachtet werden kann. So beschreibt er eine »Pluralität nicht von Attributen, sondern von Kernen ${ }^{34}$, das heißt einen » Typ von Individualisierung, der Pluralisierung zur Essenz hat «.35 Das von ihm postulierte Fehlen einer »Hyperidentität « ${ }^{36}$ steht in direkter Analogie zur dissoziativen Identität, bei welcher auch im gegenwärtigen psychiatrischen Diskurs das Festhalten am Konzept einer Ursprungspersönlichkeit zunehmend in den Hintergrund gerät. Denn es ist in der Regel nicht das chronologische Entstehen der einzelnen Alter-Persönlichkeiten, das über deren Dominanz im »System « Aufschluss gibt. Wie bei der dissoziativen Identität übernimmt auch in Welschs Vorstellung multipler Subjektivität »mal dieses, mal jenes Teilsubjekt die Führung «. ${ }^{37}$ Dieses Abwechseln der Subjektanteile wird zum einen durch eine besondere Fähigkeit zur Distanznahme beziehungsweise letztlich zur (postmodernen) Indifferenz garantiert. Auch hier liest sich Welschs Text wie die Selbstbeschreibungen sogenannter Multipler, die in zahlreichen Autobiografien ihren Niederschlag finden:

»Die Distanzierung kann ebenso der Aktivität und Durchsetzung eines der diversen Subjektanteile entspringen. Während andere Anteile sich bezüglich einer situativ nahegelegten Aktionsform indifferent oder eher zustimmend verhalten, ist ihm diese Aktionsform zuwider oder erscheint ihm als inopportun. Wenn er sich durchsetzt, wird die erwartete Handlungsform nicht übernommen, sondern abgelehnt. Eine solche Entscheidung wird also nicht durch eine übergeordnete Instanz oder eine Zentralinstanz - nicht nach einem vertikalen Schema - gefällt, sondern gleichsam horizontal durch die Subjektanteile selbst und aufgrund der besonderen Stärke der Option eines dieser Subjektanteile, welcher die Oberhand gewinnt so wie sich das nächste Mal oder in einer anderen Situation ein anderer Subjektanteil durchsetzen kann. «38

Neben dieser potenziellen Distanznahme nennt Welsch zum anderen die Übergangsfähigkeit von einem zum anderen Subjektanteil als zweites zentrales Kennzeichen beziehungsweise als zweite Bedingung einer gelingenden Vielheit, um trotz der Uneinheitlichkeit eine gewisse Kohärenz zu gewährleisten: »[D]ie Verbindung dieser diversen Identitäten erfolgt gleichsam horizontal durch Überschneidungen, Bezugnahmen und Übergänge zwischen den diversen Identitäten. Anders gesagt: Der Zusammenhang hat nicht transzendentale, sondern transversale Struktur «. ${ }^{39}$ Interaktion, wechselseitige Affizierbarkeit und Pluralitätskompetenz, das heißt die Umgangsfähigkeit mit der internen und externen Pluralität, sind Folgen einer solchen Übergangsfähigkeit. An dieser Stelle wird deutlich, dass es nicht die unbehan-

\section{Ebd.}

34 Welsch 1993, S. 161.

35 Welsch 1991, S. 180.

36 Welsch 1993, S. 178.

37 Ebd., S. 176.

38 Ebd., S. 176 f.

39 Ebd., S. 179.

Leviathan, 42. Jg., 2/2014 
delte dissoziative Identitätsstörung ist, die hier als Leitbild postmoderner Identitätstransformation gedacht werden kann, sondern jener Zwischenbereich eines (aus)therapierten Zustands, der im vorangegangenen Abschnitt als »funktionale Multiplizität « bezeichnet wurde, das heißt eines Zustands, in dem die amnestischen Barrieren zwischen den verschiedenen Alter-Persönlichkeiten weitgehend überwunden werden konnten. Erst durch das dadurch erlangte wechselseitige Bewusstsein und die darauf aufbauende strukturierte Vielheit kann das multiple Ich kooperierend als »Team " agieren; eine notwendige Voraussetzung, um als Sinnbild eines neoliberalen Leistungssubjekts dienen zu können. Es ist gerade diese Durchlässigkeit von Barrieren, die schließlich auch in der Vielheit Freiheit und Selbstbestimmung gewährleisten soll. So Welsch: »Unter Bedingungen der Pluralität ist Transversalität der Garant von Souveränität «. 40

Die von Welsch postulierte Vielheit des Subjekts wird häufig auch in neoliberalen Individualitätsdiskursen, die Subjektivität als einen unabschließbaren Gestaltungsprozess begreifen, als Chance bewertet, um die Flexibilität und produktive Anschlussfähigkeit des Individuums zu gewährleisten und womöglich zu steigern. Diese Tendenzen der Selbstoptimierung durch permanente Selbst-Neuerfindung beziehungsweise die damit einhergehende fortdauernde Anforderung nach Veränderbarkeit gilt es kritisch zu sichten.

\section{Das multiple Selbst als corporate identity}

»Die wahren Sieger leiden nicht unter der Fragmentierung, sie regt sie vielmehr an, an vielen Fronten gleichzeitig zu arbeiten; das ist Teil der Energie, die den irreversiblen Wandel antreibt. « 41

In seiner luziden Studie Das unternehmerische Selbst definiert der Soziologe Ulrich Bröckling dieses als Leitbild gegenwärtiger ökonomischer Strategien beziehungsweise als Ziel eines Subjektivierungs- sowie Verhaltensmodulierungsprogramms, welche das Individuum zum »Unternehmer in eigener Sache «42 machen sollen. Neben »klassischen " unternehmerischen Persönlichkeitsmerkmalen wie beispielsweise Eigeninitiative, Innovationsvermögen oder Risikobereitschaft legt Bröckling in seiner Analyse dabei besonderes Gewicht auf die notwendige Flexibilität und Heterogenität der einzelnen Individuen - kurzum: auf eine kreative Pluralisierung, die er nicht nur als programmatische Anforderung an einzelne Subjekte versteht, um überhaupt ein unternehmerisches Selbst werden zu können, sondern zugleich als zentrales Charakteristikum desselben.

Sieht bereits Welsch die psychische Pluralisierung des Individuums normativ geboten, wobei er dieses Gebot als praktische Pflicht zu verstehen scheint, um der Pluralität der Welt gerecht zu werden, so tritt nun in Bröcklings Lesart deutlich

40 Ebd., S. 181.

41 Sennett 1998, S. 79.

42 Bröckling 2007, S. 7. 
deren Zwangscharakter zutage. Denn das unternehmerische Selbst - und dessen bei Bröckling zugrunde liegende Multiplizität - ist kein frei zu wählender Handlungstyp unter anderen, sondern der »kategorische Imperativ der Gegenwart « beziehungsweise, so stellt dieser mit Verweis auf die Foucault'sche Gouvernementalitätstheorie fest, ein »Regierungsprogramm « ${ }^{43}$, das - gemäß dem Foucault'schen Konzept Fremd- und Selbstführung gleichermaßen umfasst. Souveränität, Flexibilität, Wahlmöglichkeit und Selbstgestaltung, bei Welsch Anfang der 1990er Jahre noch in gewissermaßen naiver Weise ungebrochen affirmierte Werte, entlarvt Bröckling nun als Zwänge eines normativen Menschenbilds, als Programm der Fremd- und Selbstgestaltung, deren "gemeinsamer Fluchtpunkt die Ausrichtung der gesamten Lebensführung am Verhaltensmodell der Entrepreneurship bildet « ${ }^{44}$ Doch Bröckling demaskiert nicht nur die Multiplizitätsanforderung der 1980er und 1990er Jahre als »Flexibilisierungsimperativ einer radikalisierten Marktökonomie « 45 , sondern erfasst in seinem Konzept des unternehmerischen Selbst auch die Radikalisierung tendenziell multipler Subjekte hin zu einem »höchst fluiden Ego, das sich in immer neuen Zusammensetzungen rekombiniert «. ${ }^{46}$ Dahingehend fährt er mit Blick auf die zunehmende »Projektisierung « des ökonomischen Handelns, ${ }^{47}$ die sich auch auf die Persönlichkeitsstruktur der Individuen überträgt, fort: »Das in den Subjektivitätstheorien der 80er- und 90er-Jahre verbreitete Schlagwort von der Patchworkidentität wäre noch zu radikalisieren: Nicht einem Flickenteppich, der, einmal genäht, sein Muster nicht mehr ändert, gleicht das sich als >Projekt-Ich` konstituierende Selbst, sondern einem Kaleidoskop, das bei jedem Schütteln ein neues Muster zeigt « ${ }^{48}$ Diese radikale Fluidität des Menschen geht mit der Anforderung einher, sich stets auf kreative Weise »neu zu erfinden « und sich Situationsveränderungen rasch anzupassen.

$\mathrm{Zu}$ einem ähnlichen Schluss kommt auch Richard Sennett in seiner Studie The corrosion of character (dt.: Der flexible Mensch ${ }^{49}$ ), wenn er konstatiert, dass der flexible Kapitalismus flexible Menschen fordere und demnach einer beständigen und einheitlichen Subjektivität, die für ihn dem Charakter eines Menschen zugrunde liegt, konsequent entgegenwirke. Denn indem die »neuen Ökonomien « auf Langfristigkeit und Dauer angelegte Strukturen konsequent abbauten und durch instabile netzwerkartige Muster ersetzten, gelinge es immer schwerer, so etwas wie Lebens-

43 Ebd., S. 9.

44 Ebd., S. 47.

45 Ebd., S. 285.

46 Ebd., S. 279.

47 Bröckling führt diesen Punkt wie folgt weiter aus: »Das unternehmerische Selbst bewegt sich schon deshalb in einem Projektkosmos, weil Entrepreneurship weder ein fixes Persönlichkeitsmerkmal noch einen erworbenen sozialen Status darstellt, sondern sich nur in actu als eine diskontinuierliche Folge unternehmerischer Handlungen, das heißt als Serie zeitlich limitierter Projekte vollzieht, die wiederum das Ergebnis sozialer Interaktionen in wechselnden Akteursnetzwerken bilden « (ebd., S. 278).

48 Ebd., S. 279.

49 Sennett 1998.

Leviathan, 42. Jg., 2/2014 
kohärenz herzustellen. Dahingehend stellt er fest: »Die Bedingungen der Zeit im neuen Kapitalismus haben einen Konflikt zwischen Charakter und Erfahrung geschaffen. Die Erfahrung einer zusammenhanglosen Zeit bedroht die Fähigkeit der Menschen, ihre Charaktere zu durchhaltbaren Erzählungen zu formen «. ${ }^{50}$ Unter dem Begriff eines flexiblen Kapitalismus versteht er hauptsächlich den diskontinuierlichen Umbau von Institutionen, die Spezialisierung von flexibler Produktion sowie die Konzentration der Macht ohne Zentralisierung. Alle drei Dynamiken zusammen fördern einen Zustand permanenter Instabilität und Ungewissheit, welcher sich auch in Subjektivierungsprozesse überträgt. So Sennett: "Das Besondere an der heutigen Ungewißheit ist die Tatsache, daß sie nicht in Verbindung mit einer drohenden historischen Katastrophe steht, sondern vielmehr mit den alltäglichen Praktiken eines vitalen Kapitalismus verwoben ist. [...] Vielleicht ist die Zerstörung des Charakters eine unvermeidliche Folge " .51 Statt folglich stabile Charaktere und langfristige soziale Kontakte zu ermöglichen, verlange das gegenwärtige kapitalistische Wirtschaftssystem ein »nachgiebiges Ich, eine Collage von Fragmenten, die sich ständig wandelt, sich immer neuen Erfahrungen öffnet - das sind die psychologischen Bedingungen, die der kurzfristigen, ungesicherten Arbeitserfahrung, flexiblen Institutionen, ständigen Risiken entsprechen «. .52

Während Welsch dank der beinahe vollständigen Ausblendung sozio-ökonomischer Implikationen die Pluralisierung des Subjekts als einen lustvollen Befreiungsprozess imaginiert, stellen Bröckling und Sennett, insbesondere letzterer ebenso etwas einseitig, die negativen Auswirkungen dieser Subjektauflösung in den Vordergrund. Ob die allgegenwärtige Angst, zu scheitern und nichts aus sich zu machen, die sowohl Bröckling als auch Sennett als Grundgefühl des neuen Kapitalismus beschreiben, der umfassende Bedeutungsverlust, die Oberflächlichkeit von Bindungen, Vereinsamung und soziale Isolation - die Folgen der allumfassenden Pluralisierung scheinen beiden Autoren wenig wünschenswert, und darüber hinaus, so Sennett, würden sie dem auf Langfristigkeit ausgerichteten menschlichen Charakter grundlegend widersprechen. Angesichts dieses Befunds verwundert es nicht, dass eine modifizierte Form von Identität, die bereits in sich Pluralisierung birgt, einen Ausweg aus dem Dilemma - oder aber im Sinne Sennetts wohl eher einen Beweis für seine Befürchtung - zu bieten scheint. Die hohe Bedeutung, die insbesondere der Flexibilität und kreativen Fragmentierung des Menschen im neoliberalen Kapitalismus zugeschrieben wird, erklärt folglich die potenzielle Leitbildfunktion dissoziativer Identität und zugleich deren Verquickung mit ökonomischen Anforderungen und Praktiken. Dahingehend stellt Bröckling selbst in einer Fußnote fest: »Es drängt sich geradezu auf, solche Vorstellungen eines pluralen Ich in Beziehung zur psychiatrischen Diskussion über >multiple Persönlichkeit`zu setzen. [...] Vielleicht erklärt sich der Aufstieg dieses Krankheitsbildes (bzw. die Konjunktur des Redens

50 Ebd., S. 37.

51 Ebd., S. 38.

52 Ebd., S. 182. 
darüber) auch aus der Parallelität der zugrunde liegenden Subjektkonzepte «.53 Bröckling deutet diesen Zusammenhang nur an, im Folgenden soll nun die angedeutete Bezugsetzung mit Rückgriff auf exemplarisch ausgewählte Texte der Gattung Autobiografie nachgezeichnet werden.

\section{Strategien der Ökonomisierung}

»Die Multiplizität brachte ganz offensichtlich die Fähigkeit mit sich, die Zeit des bewußten und halbbewußten Denkens sehr viel effizienter zu nutzen. Ich wollte nicht auf diese größere Leistungsfähigkeit verzichten, nur um so zu werden wie alle anderen. $\ll^{54}$

Zahlreiche Autobiografien von Multiplen, die seit den 1990er Jahren erscheinen, folgen demselben Erzählmuster: An ihrem Beginn steht der Entschluss der Protagonistin - die dissoziative Identitätsstörung wird zu über 90 Prozent an Frauen diagnostiziert $^{55}$-, sich in einen therapeutischen Prozess zu begeben. Die Gründe hierfür sind meist Amnesien, Depressionen, Suizidgedanken, Angstattacken und Ähnliches, doch weiß die sogenannte Gastgeberpersönlichkeit ${ }^{56}$ zu diesem Zeitpunkt noch nicht um ihre eigentliche »Erkrankung «. Die weitere Handlung besteht nun vorwiegend aus dem Therapieprozess, in welchem sich sukzessive die Diagnose dissoziative Identitätsstörung festigt, diese der Klientin nähergebracht und von jener letztlich nach einer Phase der Abwehr akzeptiert wird. Dieser Prozess geht mit der

53 Bröckling 2007, S. 70.

54 Casey, Wilson 1995 [1991], S. 361.

55 Bereits im 19. Jahrhundert wurde das doppelte Bewusstsein vorwiegend an Frauen beobachtet, was vor allem darauf zurückzuführen sein dürfte, dass dieses als spezielle Form der Hysterie eingestuft wurde, die von Beginn an als »Frauenkrankheit « galt. Für die gegenwärtige exponentiell höhere Diagnosehäufigkeit der dissoziativen Identitätsstörung bei Frauen gibt es verschiedene Erklärungsversuche. So wird beispielsweise angenommen, dass die Häufigkeit der Diagnose mit der Häufigkeit des Missbrauchs an Mädchen in Verbindung steht. Daneben vertrat bereits Cornelia Wilbur die These, dass die Dunkelziffer der betroffenen Männer deutlich höher einzustufen sei als zunächst vermutet, diese Männer jedoch eher als Delinquenten auffallen würden und weniger im psychiatrischen Umfeld. Verwiesen wird in diesem Kontext häufig auf den Fall Billy Milligan, der in den 1970er Jahren wegen mehrfacher Vergewaltigung in Ohio vor Gericht stand und als erster Angeklagter weltweit eine multiple Persönlichkeitsstörung zu seiner Verteidigung anführte.

Aus kulturwissenschaftlicher und insbesondere genderkritischer Perspektive befasst sich der 1999 von Christina von Braun und Gabriele Dietze herausgegebene Sammelband Multiple Persönlichkeit. Krankheit, Medium oder Metapher? mit der multiplen Persönlichkeitsstörung. Darin stellt beispielsweise von Braun diese in den Kontext der großen psychiatrischen »Frauenkrankheiten « (Hysterie, Anorexie), um zu untersuchen, welche »Geschlechterbilder « anhand dieser entworfen, aufgegriffen beziehungsweise modifiziert werden (von Braun, Dietze 1999).

56 Vgl. Putnam 2013 [1989], S. 81 f.

Leviathan, 42. Jg., 2/2014 
Rückerinnerung einzelner Alter-Persönlichkeiten an traumatische Kindheitserlebnisse sowie der darauf aufbauenden sukzessiven Rekonstruktion der eigenen Biografie und einer daraus entstehenden narrativen Identität einher. Ist das System der verschiedenen Alter-Persönlichkeiten zunächst chaotisch, angsteinflößend, von Amnesien durchsetzt und dadurch zumindest partiell dysfunktional, so wächst im Laufe der Therapie das Bewusstsein der einzelnen Alter-Persönlichkeiten füreinander, darauf aufbauend deren Kommunikationsmöglichkeiten untereinander und damit deren Fähigkeit zur Kooperation. Dieser Zustand, den ich eingangs als funktionale Multiplizität bezeichnet habe, entspricht weitgehend Welschs Konzept transversaler Multiplizität, und an diesem lassen sich zugleich die ökonomischen Vorteile eines solchen multiplen Subjekts im Sinne von Bröcklings unternehmerischem Selbst und Sennetts flexiblem Menschen signifikant verdeutlichen.

Hinsichtlich einer solchen Superiorität dissoziativer Störungen in Bezug auf ökonomische Anforderungen können drei Schwerpunkte unterschieden werden: die Steigerung von Effizienz, die auf einer optimierten Konzentration durch Exklusion von anderen Persönlichkeitsfragmenten basiert, die radikale Flexibilität, die in der kreativen Möglichkeit immer neu entstehender ExpertInnen gründet, und schließlich die Vielfältigkeit des Systems und das daraus resultierende Potenzial zur internen Kooperation und Netzwerkbildung. Diese Schwerpunkte lassen sich im Einzelnen an zwei Autobiografien erläutern: The flock (dt.: Ich bin viele $e^{57}$ ) von Joan Francis Casey (Pseudonym) und deren Therapeutin Lynn Wilson, eine der ersten und nach wie vor populärsten Autobiografien auf diesem Gebiet, und A fractured mind. My life with multiple personality disorder (dt.: Ich bin Robert, Wanda und $B o b b y^{58}$ ) von Robert B. Oxnam, eine der wenigen Autobiografien, die von einem Mann verfasst wurde. ${ }^{59}$ Beiden Texten gemein ist die Beschreibung eines »Zwischenzustands « der Multiplizität, der sich in ökonomischer Hinsicht als vorteilhaft erweist, auch wenn die letztliche Bewertung dieses Vorteils in den Zugängen variiert. Während in The flock eine weitgehende Affirmation dieses "Mehr " an Leistungsfähigkeit präsent ist, und das, obwohl die Protagonistin sich letzten Endes dennoch für eine Fusion der Persönlichkeitsfragmente entscheidet, mündet die gesteigerte Leistungsfähigkeit Oxnams in A fractured mind immer wieder in Burnouts und muss daher ständig justiert und optimiert werden.

57 Casey, Wilson 1995 [1991].

58 Oxnam 2008 [2005].

59 Die Selektion der Analysebeispiele richtete sich nach Kriterien der Repräsentanz, der Rezeptionspopularität, der Qualität sowie deren Diskursreflexivität. Die zur Analyse herangezogenen Beispiele wurden bislang weder einer wissenschaftlichen Betrachtung im Einzelnen noch im komparativen Vergleich unterzogen. Ähnliche Ansätze finden sich beispielsweise in den Autobiografien When rabbit howls von Truddi Chase (1990) oder Die 147 Personen, die ich bin von Liz Bijnsdorp (1994). Darüber hinaus ist die gesteigerte Funktionalität multipler Persönlichkeiten häufiger Topos zahlreicher fiktionaler Texte; siehe beispielsweise Bach 2006; Ruff 2004. 


\subsection{Effizienzsteigerung durch Exklusion}

Renee, das Erzählerinnen-Ich in The flock, ist Teil eines 24-teiligen Persönlichkeitssystems, sie ist sich dieses Umstands jedoch zu Beginn des Buchs nicht bewusst. Sie studiert Politologie, schreibt an ihrer Magisterarbeit und jobbt zugleich als Sekretärin an der Universität von Chicago, um ihr Studium zu finanzieren. Ihr Leben verlief scheinbar unbeschwert - »Die Schule war mir leichtgefallen; ich hatte nie einen Job gehabt, mit dem ich nicht klarkam. Es war immer so gewesen, daß alle mich gemocht hatten ${ }^{60}$-, gerät aber zunehmend außer Kontrolle. Es mehren sich amnestische Zustände, ihre Ehe geht in die Brüche, sie hört wiederholt innere Stimmen und findet sich schließlich auf einer Fensterbank der Universität wieder mit dem vehementen Drang zu springen. ${ }^{61}$ Von diesem Ereignis aufgeschreckt, sucht sie Hilfe in einer psychotherapeutischen Ambulanz und trifft dabei auf die Therapeutin Lynn Wilson, mit der sie eine Therapie beginnt. Mit dem Fortschreiten der Therapie treten immer neue Persönlichkeitsanteile zutage, und Wilson schöpft alsbald den Verdacht, es könne sich bei Renee um eine multiple Persönlichkeit handeln. Es entspinnt sich nach und nach eine intensive Beziehung zwischen den beiden Frauen, was immer mehr Alter-Persönlichkeiten dazu ermuntert, sich zu zeigen und ihre Biografiefragmente preiszugeben: die ernste, zurückgezogene und wissenschaftlich hochbegabte Jo, das kleine Mädchen Missy, die vorbildhafte Tochter Joan Frances, Reagan und Robin, die als Informationsspeicher dienen, die distanzierte, spöttische und zugleich künstlerisch begabte Isis, der zeitlos in der Pubertät verharrende Junge Rusty usw. Parallel zu der nun folgenden Rekonstruktion der eigenen Vergangenheit beginnt sich das Persönlichkeitssystem sukzessive als ein »Wir « - als Schwarm, wie es in der deutschen Übersetzung heißt - zu begreifen, was mit der zunehmenden Entdeckung einer bislang unbewussten inneren Ordnung einhergeht. So schreibt Renee: »[...] ich begriff, daß die Gruppe der Persönlichkeiten gar nicht so chaotisch war, wie ich anfangs gedacht hatte. Die Gruppe schien ihre ganz eigene Struktur zu haben. Ich merkte, daß sie aus völlig unterschiedlichen Persönlichkeiten bestand und daß jede von diesen Persönlichkeiten ihr eigenes Identitätsgefühl besaß«. ${ }^{62}$ Von diesem zunehmenden Wir-Gefühl und der daraus resultierenden Funktionalität ermutigt, beschließt der »Schwarm ", ein Doktorat in Harvard zu beginnen. Hier, an einer der renommiertesten Universitäten Amerikas, kann sich die Vorteilhaftigkeit eines multiplen System-Ichs und seiner spezifischen Charakteristika im Konkurrenzkampf mit KommilitonInnen in seinem vollen Umfang erweisen. Dabei ist es, wie eingangs erwähnt, gerade dasjenige, was die dissoziative Identitätsstörung als pathologisch kennzeichnet, was sich nun unter der Voraussetzung des bewussten Umgangs damit als Mehrwert herausstellt. Dies betrifft zunächst die zumindest partielle Amnesie zwischen den einzelnen Alter-Persönlichkeiten:

»[...] die Amnesie, die uns früher behindert hatte, erwies sich jetzt als Vorteil. Wir waren um ein Vielfaches produktiver als andere Leute, da sich jede Persönlichkeit ganz auf eine

60 Casey, Wilson 1995 [1991], S. 15.

61 » Spring! ‘ Ich hörte die Stimme, spürte den leisen Stoß. >Spring!« (Ebd., S. 16)

62 Ebd., S. 91.

Leviathan, 42. Jg., 2/2014 
bestimmte Aufgabe konzentrieren konnte. So arbeitete Jo beispielsweise stundenlang an irgendwelchen Referaten, ohne sich Gedanken darum zu machen, was sonst noch getan werden mußte. Wenn ich Jo beiseite schob, um meinen Lehrverpflichtungen nachzukommen, brauchte ich mir keine Sorgen wegen des Referates zu machen. Und sobald Jo sich dann wieder an die Arbeit setzte, machte sie da weiter, wo sie aufgehört hatte, ohne sich wegen der >verlorenen Zeit $<$ zu beunruhigen. Sie hatte ein richtiges Computergedächtnis, das alles speicherte, was sie mitbekam. Und ihre Konzentrationsfähigkeit wurde noch dadurch gesteigert, daß das, was zwischen ihren Bewußtseinsphasen lag, einer ebenso umfassenden Amnesie anheimfiel. «63

Im Zentrum der gesteigerten Produktivität steht folglich eine spezielle Form der Konzentration, die auf der amnestischen und damit vollständigen Exklusion der eigenen Diversität beruht. Die einzelnen Alter-Persönlichkeiten erfahren auf diese Weise zwar eine Reduktion auf ihre "Lebenswelt " sowie auf die für sie charakteristischen Begabungen und Verpflichtungen, können diesen aber dadurch zugleich in einer »Ungestörtheit " nachgehen, die dissoziierte Subjekte als ideale Modelle unternehmerischer Arbeitsteilung erscheinen lassen. Denn, so Bröckling, um »sein Humankapital zu akkumulieren, muss er [der Mensch, R.B.] sich aufspalten in ein Bündel von Vermögen und in eine Instanz, die diese Vermögen durch Tausch und Kooperation gewinnbringend verwaltet $«{ }^{64}$ Was Sennett kritisch als fragmentierende Arbeitsteilung innerhalb eines projektebasierten Kapitalismus erfasst, welche die Erfahrung eines Gesamtzusammenhangs untergrabe, entspricht folglich genuin dem Lebensalltag multipler Persönlichkeiten, was erneut deren potenzielle Leitbildfunktion verdeutlicht.

Caseys Erzählung zufolge findet dank dieser amnestischen Exklusionen bestimmter Persönlichkeitsanteile jedoch nicht nur eine Purifikation der einzelnen Kompetenzen statt, sondern damit einhergehend auch eine Effizienzsteigerung des gesamten Systems. Denn die individuellen Alter-Persönlichkeiten können nicht nur ungestört, weil ohne Ablenkung der Aufmerksamkeit durch andere Persönlichkeitsanteile und fixiert auf ein Einzelinteresse, und damit effizienter ihren jeweiligen Begabungen nachgehen, sondern das ganze System scheint nach sich abwechselnden Phasen der Produktivität und Regeneration strukturiert, sodass, während eine Persönlichkeit produktiv ist, eine andere sich erholen kann, und umgekehrt. Hierdurch mindert sich die Notwendigkeit zu schlafen, was die Effizienz des Schwarms zusätzlich steigert. So stellt die Autorin fest: »Der Schwarm brauchte nur vier bis fünf Stunden Schlaf pro Nacht. Das ließ uns viel Zeit zum Arbeiten «. ${ }^{65}$ Was hier entworfen wird, ist folglich ein leistungsstarkes System, in dem Leistungsdauer sowie die Optimierung der Arbeitskraft Hand in Hand gehen.

Dass dieser amnestische Leistungsgewinn innerhalb des Narrativs der multiplen Persönlichkeit kein Einzelfall ist, zeigt $A$ fractured mind. Hier ist es der renommierte Ostasienwissenschaftler Robert B. Oxnam, eine von elf Persönlichkeiten, der seinen

63 Ebd., S. $360 \mathrm{f}$.

64 Bröckling 2007, S. 16.

65 Ebd., S. 360. Auch Putnam verweist in seiner Studie auf die Fähigkeit vieler Multipler, ihre Energie und damit Aktivität innerhalb des Persönlichkeitssystems effizient zu verteilen, wodurch der Eindruck entstünde, sie verfügten über mehr Energie und ein geringeres Schlafbedürfnis als Durchschnittsmenschen. Vgl. Putnam 2013 [1989], S. 220 f. 
Therapieprozess beschreibt. Wie bei Casey weiß Oxnam zunächst nicht um seine Erkrankung, als er eine Therapie bei Dr. Jeffrey Smith beginnt. Er leidet unter Depressionen, unkontrollierbaren Wutausbrüchen, Alkoholsucht und einer bulimischen Essstörung. Zudem quälen ihn permanenter Leistungsdruck und Versagensängste. So beschreibt Robert seine Kindheit: »Ich war wie besessen von dem Drang, erfolgreich zu sein. War ich es, freute ich mich kurz und suchte direkt nach der nächsten Herausforderung. Der kleinste Misserfolg erfüllte mich dagegen mit Schuldgefühlen und Selbsthass « ${ }^{66}$ Die Erfolge bleiben nicht aus: Er gewinnt 17jährig die Landesmeisterschaft im Bogenschießen, hat Bestnoten in Highschool und College, studiert Asienwissenschaften in Yale mit »Rekordzeit « ${ }^{67}$, tritt im Anschluss eine Dozentenstelle für Asiatische Geschichte am Trinity College in Hartford an und landet schließlich bei der New Yorker Asiengesellschaft, deren Präsident er mit 38 Jahren wird. Mit dem massiven Arbeitsaufwand, den Robert nach außen tadellos meistert, wachsen jedoch seine gesundheitlichen Probleme. Zu den oben genannten Symptomen gesellen sich wie bei Casey Amnesien, das Hören innerer Stimmen sowie zwei Selbstmordversuche - schließlich ist es die Alkoholabhängigkeit, die ihn einen Therapeuten aufsuchen lässt, dieser stellt alsbald die Diagnose »dissoziative Identitätsstörung «. Nach dem klassischen Erzählmuster treten nun vermehrt verschiedene Alter-Persönlichkeiten zutage: der wütende Teenager Tommy, Bob, der das System zunächst nach außen vertritt, das kleine Kind Bob, die Hexe als Inbegriff von Zorn, Hass und Selbstbestrafung, der widerspenstige Junge Bobby, das allwissende Auge als »Erinnerungsdatenbank des gesamten Systems «68, Robbey, der schwierige Aufgaben im Hintergrund meistert, die spirituelle Wanda usw. Auch sie profitieren von Arbeitsteilung und wechselseitiger Exklusion:

»Unsere Subpersönlichkeiten können sich darauf konzentrieren, unabhängig voneinander neue Fähigkeiten zu erwerben und neue Einsichten zu gewinnen. Vielleicht beneiden uns normale Menschen sogar um die Arbeitsteilung, die innerhalb unseres Systems herrscht. Ich, Robert, kann mich, ohne abgelenkt zu werden, darauf konzentrieren, zu lesen, zu analysieren, Reden zu halten und zu schreiben. Bobby steht grenzenlose Energie für seinen Sport, für seinen Witz und seine Fantasie und für das Unterrichten zur Verfügung. Wanda kann sich spannenden spirituellen Herausforderungen widmen. [...] Solange einer von uns die Außenwelt bedient, können sich die anderen unabhängig um ihr Innenleben kümmern. « ${ }^{69}$

Im Gegensatz zu The flock werden in Oxnams Buch jedoch auch die Schattenseiten von optimierter Leistung, Schlafentzug und scheinbar grenzenloser Energie benannt: Bob, das dominante Außen-Ich, ist buchstäblich »ausgebrannt «70, Robbey bricht nach der Erledigung von schwierigen Aufgaben regelmäßig für mehrere Tage zusammen, und Bobby entwickelt sich unter dem enormen Leistungsdruck zum Kleinkriminellen. Als Bobs Burnout-Syndrom nicht mehr weiter zu ignorieren ist, beschließt er, mit einer anderen Alter-Persönlichkeit, dem engagierten und (noch) energiege-

66 Oxnam 2008 [2005], S. 22.

67 Ebd., S. 28.

68 Ebd., S. 166.

69 Ebd., S. 230.

70 Ebd., S. 79.

Leviathan, 42. Jg., 2/2014 
ladenen Robert, zu fusionieren, das heißt ihm die Führung des Persönlichkeitssystems zu überlassen und dabei in Form einer Teil-Integration in ihm "aufzugehen ". Bob kommentiert den Vorgang folgendermaßen: »Für jemanden, der nicht an dieser Persönlichkeitsstörung leidet, hört es sich vielleicht verrückt an, aber die Krankheit eröffnete mir die Möglichkeit, meine Fähigkeiten und mein Gedächtnis an jemanden weiterzugeben und beruflich keine Verantwortung mehr tragen zu müssen. Es war wirklich nicht wie sterben, sondern eher wie eine Reinkarnation " ${ }^{71}$ Für die Fusion werden wie bei tatsächlichen Unternehmenszusammenführungen Verträge ausgehandelt, die beide Parteien absichern sollen. So verlangt Bob beispielsweise die vorläufige Beibehaltung seiner Namenskurzform in beruflichen Angelegenheiten, quasi als Trademark, und Robert fordert langfristig die Kündigung als Leiter der AsienGesellschaft sowie "alleinige Entscheidungsbefugnis «72 bei der Wahl einer neuen beruflichen Tätigkeit. Zuletzt führt Bob eine »Rücktrittsklausel «73 ein, sollte Robert die Abmachung nicht einhalten: »Es war ein Deal: Ich war der müde gewordene, alte Geschäftsführer, der in Frührente ging, aber im Vorstand blieb. Außerdem konnte ich Einspruch erheben, falls der neue Chef mein Vermächtnis ablehnte «. ${ }^{74}$ Auffallend an den Textbeispielen ist neben dem inhaltlichen Bezug auf Leistung und Effizienz auch die Wortwahl, die das multiple Persönlichkeitssystem gezielt in den Kontext ökonomischen Handelns rückt. Doch auch Roberts berufliche Neuorientierung - er tritt zunächst einen Beraterposten in einer privaten Investmentbank an und im Anschluss Dozentenstellen für Asiatische Studien an der Columbia University und am Williams College - mündet alsbald erneut in einem Burnout, denn die »Robert-Bob-Robbey-Triade [war] täglich vierzehn Stunden oder noch länger im Einsatz $[\ldots] \ll{ }^{75}$ Diese Phasen von Höchstleistung und Erschöpfung setzen sich in der Folge weiter fort und bedingen eine mehrfache Neuausrichtung des Systems. Doch trotz dieser wiederholten "Störfälle " gelangt Oxnam letztlich zu der Überzeugung: »Ich glaube, dass mein beruflicher Erfolg nicht trotz meiner Störung möglich war, sondern, ganz im Gegenteil, gerade wegen ihr «. ${ }^{76}$ Es ist folglich die Dissoziation, welche die ökonomische Leistungsfähigkeit bedingt.

71 Ebd., S. 111.

72 Ebd., S. 113.

73 Ebd., S. 114.

74 Ebd., S. $114 \mathrm{f}$.

75 Ebd., S. $184 \mathrm{f}$.

76 Ebd., S. 173. 


\subsection{Flexible Spezialisierung durch Überforderung}

»Es erschien mir ganz normal, dass ich plötzlich Chinesisch konnte, so als hätte ich eine neue Software auf meinem Computer installiert. «77

Flexibilität beziehungsweise Biegsamkeit gehören nach Sennett und Bröckling zu den zentralen Merkmalen des neoliberalen Leistungssubjekts. Auch hier ist es wiederum das, was die dissoziative Identitätsstörung als pathologisch kennzeichnet, was ihr angesichts dieser Flexibilitätsanforderung zugleich Vorteile verschafft. Denn das System multipler Persönlichkeiten beruht in seinem Kern auf der Notwendigkeit, zunächst die Primärpersönlichkeit und später alle weiteren Persönlichkeiten vor Überforderung zu schützen. Sind es zu Beginn der Störung traumatische Erlebnisse, die eine solche Überforderung und in der Folge eine Dissoziation hervorrufen, so tritt mit der Zeit eine gewisse Verselbstständigung des Dissoziationsprozesses ein, sodass auch herkömmliche Stresssituationen oder herausfordernde Umstellungen die Entstehung einer neuen Alter-Persönlichkeit auslösen können; ein Umstand, der sich in einer bisweilen auf mehrere hundert ansteigenden Zahl an Alters niederschlägt. Die Überforderungen durch mehr oder weniger alltägliche Problemstellungen kommen umso mehr zum Tragen, als einzelne Alters in der Regel nicht über dieselbe Komplexität wie nicht-dissoziierte Menschen verfügen. Das ist darauf zurückzuführen, dass ihnen zum einen nicht dieselbe Quantität an Zeit zur Verfügung steht, um an der Außenwelt zu wachsen. Zum anderen werden sie bereits bei ihrem Entstehen für spezifische Situationen und Anforderungen "geschaffen ", denen sie in der Folge beinahe typologisch entsprechen, ${ }^{78}$ was alternative Fertigkeiten bis zu einem gewissen Grad ausschließt. Die dissoziative Identitätsstörung ist folglich ihrem Wesen nach durch eine ausgeprägte Inflexibilität der einzelnen AlterPersönlichkeiten gekennzeichnet. Doch gerade diese Inflexibilität birgt die Möglichkeit einer nahezu uneingeschränkten Einsatzfähigkeit, da neue Herausforderungen nicht eine mehr oder weniger zeitaufwendige Anpassung voraussetzen, sondern die Kreation eines besonderen Individuums, das optimal auf diese neue Situation abgestimmt scheint, das heißt eines Experten. Das Fehlen von Ambiguitätstoleranz, also die Fähigkeit, mit Unsicherheiten umgehen zu können, wird durch Dissoziation kompensiert und macht das multiple Subjekt auf diesem Wege höchst anpassungsfähig. Dahingehend stellt Renee fest: »Ich hatte keinen eigenen inneren Kern zu schützen. Ich konnte mich schnell und mühelos verändern, wie ein Chamäleon $[\ldots] \ll{ }^{79}$ Die Aushöhlung des Charakters, die Sennett innerhalb der neoliberalen

77 Ebd., S. 118.

78 Putnam unterscheidet unter anderem Gastgeber-Persönlichkeiten, Kind-Persönlichkeiten, Beschützer- und Helfer-Persönlichkeiten, Erinnerungsträger, Verwalter-Persönlichkeiten, Innere Selbst-Helfer, promiskuitive Persönlichkeiten, Persönlichkeiten mit besonderen Talenten und Fähigkeiten, schmerzabgestumpfte und -unempfindliche Persönlichkeiten, autistische und behinderte Persönlichkeiten, Persönlichkeiten mit Substanzmissbrauch, Alter-Persönlichkeiten des anderen Geschlechts, Verfolger-Persönlichkeiten und suizidale Persönlichkeiten. Vgl. Putnam 2013 [1989], S. 135 f.

79 Casey, Wilson 1995 [1991], S. 196.

Leviathan, 42. Jg., 2/2014 
Marktökonomie wahrzunehmen vermeint, geht in diesen Fällen angesichts einer solchen Kernlosigkeit respektive Hohlheit des Ichs ins Leere, da sie bereits vollständig umgesetzt ist. Auf einer ganz anderen Ebene scheint sich daher angesichts der stetig steigenden Zahl diagnostizierter Fälle dissoziierter Identität Sennetts Klage als Zeitbefund zu bestätigen. Und angesichts der vergleichsweise ungezügelten Form der freien Marktwirtschaft ist es möglicherweise auch kein Zufall, dass die dissoziative Identitätsstörung in erster Linie innerhalb der USA auftritt. Es lässt sich also fragen, ob die Dissoziation als Störung im Zusammenhang mit potenziell schädigenden Wirtschaftsstrukturen oder aber als optimierte Anpassung und damit als Leitbild zu bewerten ist, das heißt, ob angesichts der Ökonomisierung alternativer Subjektivitäten hinsichtlich der dissoziativen Identitätsstörung überhaupt noch von der Notwendigkeit der Behebung einer Devianz auszugehen ist oder ob nicht sogar, um es provokant zu formulieren, eine »kulturevolutionäre « Überlegenheit vorliegt. Eine solche Lesart würde jedoch die im Moment etablierte psychiatrische Ätiologie der dissoziativen Identitätsstörung, nämlich wiederholte schwere Traumata in der frühen Kindheit, ignorieren - und das wäre problematisch. Es lässt sich jedoch denken, dass die gesteigerten Leistungs- und Flexibilitätserwartungen der neoliberalen Ökonomie wenn auch nicht kausal, so doch in der positiven Fremd- und Selbstbewertung der "Störung " und damit im Umgang mit dieser eine wesentliche Rolle spielen. So könnten beispielsweise die Entscheidung vieler Multipler, ihren Zustand einer funktionalen Multiplizität beizubehalten, oder auch die Faszination für das Phänomen innerhalb und außerhalb der Forschung auf diese Korrelation mit ökonomischen Anforderungen zurückzuführen sein. ${ }^{80}$

Die ökonomische Verwertbarkeit dissoziativer Flexibilität stellt auch die Therapeutin Lynn Wilson in den Vordergrund, wenn sie den Dissoziationsprozess ihrer Patientin in einem in die Autobiografie eingefügten Tagebucheintrag beschreibt:

\begin{abstract}
»Bald darauf erwarb Renee durch ihr geschicktes Auftreten bei Vorstellungsgesprächen eine begehrte Praktikumsstelle bei der Gesetzgebung und später einen bezahlten Job bei der Pressestelle. Durch ihre Arbeit fühlte Renee sich jedoch überfordert. Sie sah keine Möglichkeit, wie sie alle zufriedenstellen könnte. Cassandra wurde nach dem Modell einer Rechtsanwältin geschaffen. Cassandra arbeitete produktiv und professionell, sie war herzlich und kompetent, ohne Renees ausgeprägtes Bedürfnis zu haben, es allen recht zu machen. Doug, eine männliche Persönlichkeit, die sich an einem Wahlkampfmanager orientierte, entstand zur gleichen Zeit wie Cassandra. Doug erzählte mir, Cassandra sei ganz gut allein zurechtgekommen, aber er sei gebraucht worden, weil wir sin einer Männerwelt leben. Abgesehen davon, daß er Golf spielen lernte, ist nicht klar, was Doug eigentlich machte, um >den Mädchen mal ein bißchen auf die Sprünge zu helfen « ${ }^{81}$
\end{abstract}

80 Publizierte Fallberichte seit dem 19. Jahrhundert machen deutlich, dass die Faszination an der multiplen Persönlichkeit zu einem nicht unbeträchtlichen Teil auf den Fähigkeiten und oftmals erstaunlichen Unterschieden zwischen den Alter-Persönlichkeiten basiert. Auf diese Unterschiede fokussieren auch neuere Forschungen zur dissoziativen Identitätsstörung, die häufig neurobiologische Faktoren untersuchen.

81 Casey, Wilson 1995 [1991], S. 190 f. Der Begriff "geschaffen « suggeriert die aktive und gezielte Hervorbringung neuer Alter-Persönlichkeiten. Wie ein solcher Produktionsprozess und insbesondere dessen scheinbare Effektivität jedoch genau vorzustellen wären, bleibt unausgeführt. 
Dass diese Funktionalität der Dissoziation alle Bereiche des Lebens umfasst, zeigt Renees lakonischer Kommentar: »Charlene und Honey entstanden, weil ich nie so ganz dahinterkam, wie ich Keith eine gute Ehefrau sein könne «. .82

Auch die Entstehung von Bob, Robert Oxnams dominanter Außenpersönlichkeit, ist auf eine ähnliche Überforderungssituation zurückzuführen. So erzählt Robbey, der quasi von Bob in dieser Funktion abgelöst wurde:

»In meinem zweiten Studienjahr hatte ich mich für einen Rhetorik-Kurs eingetragen. In den ersten beiden Semesterwochen gab der Professor Tipps, wie wir die Reden in der Öffentlichkeit halten sollten. Dann forderte er uns Studenten auf, jede Stunde kurze Referate zu halten, die er und die Gruppe anschließend beurteilten. Ich geriet in Panik. [...] Ich hatte furchtbare Angst davor, ein Referat zu halten. Nachdem ich alle Tricks über das Sprechen in der Öffentlichkeit erfahren hatte, strich ich den Kurs aus meinem Semesterplan. Papa war fassungslos, als ich es ihm erzählte. [...] Ich weinte nicht - ich weine nie -, aber ich war sehr traurig. Und in diesem Moment tauchte jemand anderes in der Burg auf. Jemand, der Dinge in der Öffentlichkeit tun konnte: Reden halten, Besprechungen leiten, unterrichten, im Fernsehen auftreten. ${ }^{83}$

Dieser Entstehungsprozess immer neuer Fachbegabungen korreliert mit dem, was Sennett die flexible Spezialisierung innerhalb flexibler Wirtschaftsordnungen nennt. Denn wichtigster Bestandteil dieses neuen Produktionsprozesses ist es, »erstarrte Unternehmensformen zugunsten der Innovation aufzubrechen und die Binnenstruktur von Unternehmen durch die wechselnden Forderungen der Außenwelt bestimmen zu lassen: all dies erfordert die Akzeptanz entschiedenen, abrupten, irreversiblen Wandels $«{ }^{84} \mathrm{Im}$ Vorteil ist, wer umstandslos bisher eingeschlagene Wege wieder verlassen und neue Terrains vorbehaltlos auftun kann. Auch das System multipler Persönlichkeiten reagiert in hohem Maße flexibel auf wechselnde Anforderungen der Außenwelt, indem es deren Leistungsansprüche und die diesen inhärente Dynamik aus Belohnung und Bestrafung, personifiziert in der Figur des enttäuschten Vaters, verinnerlicht. Diese Fähigkeit zur funktionellen Adaption ist ein grundlegendes Charakteristikum der dissoziativen Identitätsstörung, auf welches sowohl in der klinisch-therapeutischen Literatur als auch in zahlreichen Autobiografien verwiesen wird. Dabei stehen nicht immer, so wie in den ausgewählten Texten, berufliche Kontexte im Vordergrund, sondern häufig das (Über-)Leben innerhalb eines als bedrohlich empfundenen Familiensystems. Doch in beiden Fällen wird auf die erstaunlichen Fähigkeiten der Betroffenen verwiesen, auf eine instabile und unberechenbare Umgebung durch die Herausbildung immer neuer Identitäten höchst anpassungsfähig zu reagieren. Aus der Not der Überforderung beziehungsweise der Angst, nicht genügen zu können, wird dadurch aus ökonomischer Sicht gewissermaßen eine Tugend, denn die Inflexibilität der einzelnen Alter-Persönlich-

82 Ebd., S. 187.

83 Oxnam 2008 [2005], S. 79. Betroffene der dissoziativen Identitätsstörung imaginieren häufig ihr inneres System in Form eines Gebäudes, in dem die verschiedenen Alter-Persönlichkeiten wohnen. Im Falle Oxnams handelt es sich dabei um eine Burg.

84 Sennett 1998, S. 65.

Leviathan, 42. Jg., 2/2014 
keit eröffnet dem System zugleich scheinbar unbegrenzte Möglichkeiten der Belastung. Auf diese Potenzialität nimmt auch Lynn Wilson Bezug:

»Als ich das erste Mal hörte, wie die Kendra Persönlichkeit (die Renee rettet, wenn sie sich zu viel vorgenommen hat) sagte: ’Wir können alles! ‘, hielt ich das für übertrieben. Jetzt sehe ich, daß es, gemessen an den meisten Leuten, stimmt. In vieler Hinsicht können die Persönlichkeiten tatsächlich alles, was sie in Angriff nehmen, solange nicht erwartet wird, daß eine Persönlichkeit allein über längere Zeit alles übernimmt. « ${ }^{85}$

Tatsächlich umfassen die geschilderten Fähigkeiten beziehungsweise Fertigkeiten einzelner Alters häufig ein fotografisches Gedächtnis, künstlerische Talente, Schmerzunempfindlichkeit, ein verringertes Schlafbedürfnis, enorme körperliche Kräfte, sportliche Fähigkeiten, erhöhte Intelligenz und Ähnliches. ${ }^{86}$ Was in dem Konzept der dissoziativen Identität folglich imaginiert wird, ist das Modell eines im höchsten Maße biegsamen und zugleich fähigen Subjekts - eine, so Welsch, »quasi kernlose, eine rein aus der Vielheit von Möglichkeiten bestehende Identität ${ }^{87}$-, das in seinen fortwährenden Zyklen aus Expansion und Fusion zugleich durch eine radikale Unabgeschlossenheit gekennzeichnet ist. In dieser nie einzuholenden Abschließbarkeit korreliert die dissoziative Identitätsstörung einmal mehr mit Bröcklings Beschreibung des unternehmerischen Selbst, denn: "Anders als das traditionelle Disziplinarsubjekt, das niemals aufhört anzufangen, wird der Unternehmer in eigener Sache nie mit irgendetwas fertig. Permanente Weiterbildung, lebenslanges Lernen, persönliches Wachstum - die Selbstoptimierungsimperative implizieren die Nötigung zur kontinuierlichen Verbesserung «. 88

Nicht zuletzt entspricht das multiple Selbst in dieser stetigen Selbstkreation einer weiteren zentralen Bedingung unternehmerischer Superiorität - jener der Kreativität. So wird die dissoziative Identitätsstörung in ihrer Entstehung sowie in der immer weiter fortschreitenden Hervorbringung von Alter-Persönlichkeiten sowohl in EgoDokumenten Betroffener als auch im psychiatrischen Fachdiskurs häufig in den Kontext einer besonderen Kreativität gestellt, und der Tenor in der Multiplendebatte lautet daher: »Multiple Persönlichkeit, so unverständlich und furchterregend dies für viele, für Fachleute wie für die Allgemeinheit, auch sein mag, ist die Reaktion eines kreativen Geistes [...] «. ${ }^{89}$ Umgekehrt zeichnen sich schöpferische Menschen, so Bröckling, gerade dadurch aus, dass sie möglichst gegensätzliche Eigenschaften in sich verkörpern. Denn das »kreative Subjekt (zu dem sich ja alle heranbilden sollen) kann immer auch anders und befindet sich in fortwährender Bewegung, um

85 Casey, Wilson 1995 [1991], S. 189.

86 Auch Putnam verweist in seinem Handbuch zur dissoziativen Identitätsstörung auf die Außergewöhnlichkeit und die vielseitigen Talente vieler Betroffener, wie sie insbesondere bei Persönlichkeitsfragmenten, die eigens für bestimmte Aufgaben entstanden sind, zutage treten. Vgl. Putnam 2013 [1989], S. 141. Zugleich schreibt er insbesondere den sogenannten Verwalter-Persönlichkeiten ein hohes Maß an beruflicher Kompetenz zu, was oftmals zu verantwortungsvollen und hochrangigen Positionen innerhalb der Arbeitswelt führe. Vgl. ebd., S. 140.

87 Welsch 1991, S. 180.

88 Bröckling 2007, S. $71 \mathrm{f}$.

89 Phillips 1992 [1987], S. 13. 
die widersprüchlichen Anforderungen auszutarieren $" .{ }^{90}$ Kreativität und Heterogenität, zwei grundlegende Charakteristika des unternehmerischen Selbst, finden folglich in der Dissoziation exemplarischen Ausdruck.

\subsection{Kooperation statt Integration}

»In meinem Innern herrschte eine perfekte Symbiose, nur war sie mir nicht bewusst. «91

Das multiple Subjekt ist eine flexible Organisation, ein mittelpunktloses Netz, das durch Dezentralisierung gekennzeichnet ist, nach Sennett neben der flexiblen Spezialisierung und der diskontinuierlichen Umstrukturierung von Institutionen die dritte zentrale Eigenschaft eines flexiblen Kapitalismus. Es besteht folglich bloß aus »Verbindungen und Knoten des Netzes ${ }^{92}$, ohne jedoch ein Zentrum aufzuweisen. Diese dezentrierte Struktur der multiplen Persönlichkeit wird zunächst als Bedrohung beziehungsweise als dysfunktional wahrgenommen. Ob metaphorisch als zerbrochenes Puzzle, als Chaos, als auswegloses Labyrinth, als zersplittert und fragmentiert gedacht, das identitäre Gefüge multipler Persönlichkeit ist Inbegriff einer Unordnung, die nicht nur die Kontrolle über gegenwärtiges Verhalten, sondern auch den geordneten Zugang zu Erinnerungen und damit zur eigenen Biografie betrifft. Ziel der Therapie ist es nun, wie ausgeführt, dieses Chaos zu ordnen, das heißt die in Unordnung geratenen Persönlichkeitsfragmente zu einem »kooperativen System «, einem »strukturierten Schwarm « zu fügen. Hierfür ist es zentral, »die Kommunikationskanäle wieder zu öffnen und den Dialog innerhalb des Persönlichkeitssystems zu reaktivieren $" .{ }^{93}$ Für die Förderung der inneren Kommunikation schlägt Putnam, einer der führenden Experten auf diesem Gebiet, mehrere Stufen vor. Tritt zunächst der Therapeut als Vermittler zwischen den einzelnen Persönlichkeiten auf, so werden diese in einem zweiten Schritt dazu angehalten, sich gegenseitig Nachrichten in einem Notizbuch oder Ähnlichem zu hinterlassen. In einem fortgeschrittenen Stadium der Therapie kommt es schließlich zu inneren Gesprächen, zunächst in Dialogform und letztlich auch in Form von Gruppendiskussionen, die es moderierend zu leiten gilt. Auch in diesem Bereich der inneren Kommunikation zeigen sich signifikante Parallelen zwischen dem psychiatrischen Narrativ der multiplen Persönlichkeit und dem ökonomischen des unternehmerischen Selbst, wie das folgende Zitat verdeutlicht:

»Sind alle Persönlichkeitsteile voll im Einsatz? Arbeitet jeder Teil an der Stelle, wo er seinen Fähigkeiten entsprechend optimale Ergebnisse erzielen kann? Arbeiten die Teile alle gut zusammen, oder gibt es Konkurrenz und Kompetenzgerangel? Müssen Sie befürchten, daß einige Teile am Ende gar die sinnere Kündigung`vollzogen haben? Kennen sich überhaupt

90 Bröckling 2007, S. 167. Bröckling bezieht sich auf verschiedene Positionen aus der Kreativitätsforschung wie beispielsweise jene von Mihaly Czikszentmihalyi, Carl Rogers oder Joy P. Guilford.

91 Oxnam 2008 [2005], S. 80.

92 Sennett 1998, S. 71.

93 Putnam 2013 [1989], S. 186.

Leviathan, 42. Jg., 2/2014 
alle Teile untereinander, oder fühlen Sie sich eher als das Opfer zusammenhangloser Einzelteile? «94

Was sich wie ein Fragebogen aus dem Kontext der multiplen Persönlichkeitsstörung liest, stammt aus dem Ratgeber Coach Yourself (1991), den Bröckling als paradigmatische Anleitung für die Herausbildung und Verwaltung einer sogenannten IchAG zitiert. Denn um eine funktionale Heterogenität des Subjekts zu gewährleisten, so stellt Bröckling fest, ist inneres Teamwork vonnöten. Konkretisierend nennt er Planung und kontinuierliches Controlling, koordinierende Moderation der disparaten Wünsche sowie (Selbst-)Enthusiasmierung als Eckpfeiler einer solchen kommunikativen Zusammenarbeit. ${ }^{95}$ Dabei soll innerhalb eines multiplen Projekt-Ichs auch die Führung einzelner Projektanteile, so macht obiges Zitat deutlich, nach dem Vorbild der Unternehmensführung beziehungsweise des Projektmanagements vonstatten gehen. Denn um »zum Unternehmer seiner selbst zu werden, liegt es nahe, sich selbst als Unternehmen zu imaginieren " ${ }^{96}$

Auch in The flock finden zwecks Moderation beziehungsweise Koordination der heterogenen Wünsche und Stärken wiederholt innere »Konferenzschaltung[en] «97 statt, in denen oben zitierte Fragen eine Klärung erfahren sollen. Entsprechend der vermeintlichen Fähigkeit dissoziativer Identitäten zur spezifischen Flexibilisierung entsteht hierfür eine eigene Expertin, welche die Kommunikation anleiten soll:

»Ende Oktober fand ich eines Morgens beim Aufwachen mehrere Blätter mit einem maschinengeschriebenen Gesprächsprotokoll: das Produkt einer ganz neuen Form schwarminterner Kommunikation. [...] Die Persönlichkeit, von der diese neue Fähigkeit ausging, nannte sich Unity. [...] Sie behauptete, sie sei einzig und allein dazu da, uns zu helfen, effizienter miteinander zu kommunizieren und enger zusammenzurücken. [...] Unity initiierte und leitete Diskussionen in einer selbstinduzierten hypnotischen Trance. Da ihr klar war, daß zumindest für einige von uns das bewußte Wissen um diese mitternächtlichen Konferenzen wichtig war, bat Unity die praktische Karen-Persönlichkeit, während der Besprechungen Protokoll zu führen. «98

Ziel dieser Trance-Konferenzen ist es, die Kooperationsfähigkeit des Schwarms und damit seine Leistungsfähigkeit zu steigern, wozu dieser sich trotz seiner Heterogenität als Team begreifen muss. Denn da ein »Projekt Ich sich seine Mitarbeiter weder aussuchen noch sie bei unbefriedigender Leistung kurzerhand entlassen kann «, so führt auch Bröckling aus, »bleibt ihm nichts anderes übrig, als die heterogenen, möglicherweise widerstreitenden Elemente miteinander zu versöhnen. Moralisierung ist dabei kontraproduktiv: Es gibt keine guten und schlechten Persönlichkeitsanteile, sondern nur ein gut oder schlecht kooperierendes Team $" .{ }^{99}$ In diesem Sinne

94 Besser-Siegmund, Siegmund 1991, S. 73 f., zitiert nach Bröckling 2007, S. 69 f.

95 Vgl. Bröckling 2007, S. 280.

96 Ebd., S. 69.

97 Casey, Wilson 1995 [1991], S. 293.

98 Ebd., S. 370.

99 Bröckling 2007, S. 281. Unklar bleibt in diesem Entwurf, welcher Subjektanteil die Führung des Teams übernehmen soll, das heißt, ob das Persönlichkeitssystem hierarchisch strukturiert oder aber im Sinne Welschs horizontal und somit in den Führungspositionen variabel zu denken ist. 
stellt er pointiert fest: »Identität ist in diesem Persönlichkeitsmodell Corporate Identity «. 100

Kooperation statt Integration ist auch die Devise zahlreicher Autobiografien von Multiplen, wie Renees Feststellung in The flock exemplarisch verdeutlicht: »Wir kehrten mit dem Gefühl nach Harvard zurück, daß wir auf eine ganz neue Weise zusammengerückt waren. Wir wurden uns immer sicherer, daß Kooperation, nicht Integration für uns das realistische Ziel war. Wir wollten unbedingt beweisen, daß wir auch als Gruppe mit dem Leben zurechtkamen «. ${ }^{101}$ Dieser Anspruch des "Zurechtkommens « wird in der Folge als Euphemismus entlarvt, denn einmal mehr erweist sich die Gruppenstruktur in der Konkurrenz mit KommilitonInnen als signifikanter Vorteil. Dabei sind es nicht einzelne Persönlichkeiten, sondern die Synergien des gesamten Systems, das heißt des Netzwerks, die den ökonomischen Mehrwert bedingen. Dies jedoch nur durch die strukturierte Zusammenarbeit des Schwarms. Erst mittels Absprachen und gezielter Arbeitsteilung kann sich dessen Superiorität vollends entfalten, wie das nachfolgende Zitat veranschaulicht:

»Die Allianz war locker genug, daß Kendra, Isis und ich zwar kooperierten, aber autonome Persönlichkeiten blieben. Kendra und ich liebten beide die juristischen Lehrveranstaltungen. Wir bereiteten gemeinsam die Fälle vor und trieben noch zusätzliche Studien in der juristischen Bibliothek [...]. Wenn wir im Seminar etwas referieren sollten, übernahm ich das Reden, wobei ich jedoch innerlich die Ohren spitzte, um Kendras Anregungen und Bemerkungen aufzunehmen. Sie speicherte beim Lesen vieles, was mir entging. Wenn andere Studenten referierten oder der Professor etwas vortrug, machte ich mit der rechten Hand Notizen auf meinem Block, während ich in der linken den Kommentar hielt. Dann kam es oft vor, daß Kendra, die mit beiden Händen gleich geschickt war, mit der linken Anmerkungen in den Kommentar schrieb, während ich gleichzeitig mit rechts Notizen machte. «102

In ähnlicher Weise beschreibt und bewertet auch Oxnam seine Persönlichkeitsstruktur: »[...] mir wurde klar, dass unsere innere Struktur für mich als Dozent einen Vorteil hatte. Wenn multiple Subpersönlichkeiten innerlich zusammenarbeiten, können sie in einem multikulturellen Kontext besser agieren. Während einer von uns sprach, hörten die beiden anderen zu und beobachteten. Das machte es leichter, sich in andere einzufühlen «. ${ }^{103}$ Die Fähigkeit zum »Multitasking «, ein weiteres Schlagwort ökonomischer Funktionalität, gewinnt in dieser Form der simultanen Arbeitsteilung eine neue Entsprechung. Die Möglichkeit zur inneren Kommunikation und in der Folge zur Kooperation vereint folglich die Vorteile der Effizienz durch Exklusion und der spezifischen Flexibilisierung, indem sie diese steuerbar und damit planbar macht. Dissoziation avanciert somit letzten Endes in diesen Erzählungen zu einem verfügbaren Instrument, das sich vielseitig für ökonomische Zwecke einsetzen lässt. Es ist daher innerhalb der autobiografischen Narration der multiplen Persönlichkeit nur folgerichtig, wenn Renee letztlich zu der Einsicht gelangt: »Vielleicht sind zwei oder mehr Köpfe ja tatsächlich besser als einer «. ${ }^{104}$

100 Ebd., S. 70.

101 Casey, Wilson 1995 [1991], S. 360.

102 Ebd., S. 312.

103 Oxnam 2008 [2005], S. 249.

104 Casey, Wilson 1995 [1991], S. 312.

Leviathan, 42. Jg., 2/2014 


\section{Schlussbemerkungen}

Wie gezeigt wurde, erfolgt die Ökonomisierung devianter Subjektivitäten in mehreren Richtungen. Auf der einen Seite verlangen neue Ökonomien alternative Subjekte, die versprechen, der Pluralitätsanforderung der Welt gerecht zu werden. Hierbei kommt es häufig zur Vereinnahmung pluraler Subjektformen und Differenzkonzepte, wie sie in verschiedenen philosophischen Strömungen seit dem Poststrukturalismus erörtert werden. ${ }^{105}$ Diese differenzphilosophischen, postmodernen, queertheoretischen und postkolonialen Auseinandersetzungen wiederum nehmen wiederholt, wie exemplarisch an Welsch dargelegt, Anleihen an psycho-sozialen Störungsbildern, die symbolisch aufgeladen werden. ${ }^{106}$ Darüber hinaus bedingen künstlerische sowie populär-kulturelle Beschäftigungen mit dem Phänomen die mediale Präsenz und zugleich das Anhalten der Faszination desselben. ${ }^{107}$

Nicht zuletzt sind insbesondere autobiografischen Dokumenten Leistungsimperative und das Streben, diesen in optimierter Weise zu entsprechen, eingeschrieben. Dabei werden gerade die Charakteristika der Dissoziation, wie anhand zweier Autobiografien exemplarisch dargelegt wurde, als vermeintliche Vorteile ökonomisch erfolgreichen Verhaltens präsentiert. Die Summe dieser nahezu unbegrenzten Fertigkeiten und Fähigkeiten, welche die dissoziative Identitätsstörung den Berichten Betroffener zufolge bedingt, gemahnt bisweilen an ein weiteres amerikanisches "Markenprodukt « - den Superhelden. In beiden Fällen dient die Überlegenheit in der Regel der Kompensation eines Mangels beziehungsweise - präziser - dieser vermag dadurch kompensiert zu werden, dass dessen Devianz als Potenzial entdeckt wird. Dieser Umschreibungsprozess, der in erster Linie von Betroffenen selbst vollzogen wird, dient der Legitimation »in Unordnung geratener « Subjekte innerhalb einer auf Funktionalität ausgerichteten Gesellschaftsstruktur. So stellt Renee fest:

105 Vgl. hierzu beispielsweise Ha 2005 oder Engel 2009.

106 Vgl. hierzu beispielsweise Deleuze, Guattari 1977 [1972]. Es kann aus wissenstheoretischer Perspektive gefragt werden, wie veränderte Vorstellungen vom Ich, von Identität und Subjektivität die Möglichkeit, multiple Persönlichkeitstypen überhaupt als Störungsbild zu »entdecken «, gefördert haben. So fällt auf, dass beide Höhepunkte des psychiatrischen Phänomens mit philosophischen »Krisen des Subjekts « einhergegangen sind, in denen Erkenntnisparadigmen über das Ich aufgegeben beziehungsweise radikal verändert wurden. Umgekehrt blieben jedoch auch letztere von der humanwissenschaftlichen Entdeckung der Persönlichkeitsspaltung nicht unbeeinflusst. So finden sich etwa seit den 1990er Jahren vermehrt philosophische Auseinandersetzungen mit multipler Persönlichkeit, beispielsweise bei Daniel C. Dennett und Nicolas Humphrey (1989) oder Stephen Braude (1991), die das Störungsbild in ihre bewusstseinsphilosophischen Studien integrieren und dabei der Frage nachgehen, ob es philosophisch-analytisch möglich ist, mehrere apperzeptive Zentren des Bewusstseins innerhalb einer Person zu denken. In diese Richtung geht auch der analytische Ansatz von Logi Gunnarsson (2010), im Zuge dessen er personale Identität neu zu verorten versucht.

107 Spätestens mit der US-amerikanischen Dramedy United States of Tara (2009-2011), die das turbulente Leben der an einer dissoziativen Identitätsstörung leidenden Tara (Toni Colette) auf humorvolle Art zeigt, ist das Phänomen Teil der Populärkultur geworden. 
»Es ist doch gerade unsere Funktionstüchtigkeit, die es uns ermöglicht, mit dem Wahnsinn fertig zu werden «. ${ }^{108}$ Nicht zuletzt stellt diese Stilisierung zum souveränen Leistungssubjekt auch einen Versuch der Selbstermächtigung dar, welcher der dissoziativen Auflösung von Identität zum Trotz auch als eine Form der Resubjektivierung verstanden werden kann, das heißt als eine Aktualisierung beziehungsweise Rekonstituierung traditionell subjektiver Attribute wie Souveränität, Selbstverwirklichung, Effizienz und kreative Selbstgestaltung aus der (post)modernen Krise des Subjekts. Diese Resubjektivierung ereignet sich jedoch gerade nicht, so zeigen sowohl die zitierten Autobiografien als auch Welschs Aufsätze, in einer normalisierenden Abgrenzung von dem sogenannten Devianten, sondern indem das vormals Deviante nun vielmehr selbst zum Konstituens gelingender Subjektivität avanciert.

Wenn Sennett schließlich feststellt, »[d]ie Fähigkeit, sich von der eigenen Vergangenheit zu lösen und Fragmentierung zu akzeptieren, ist der herausragende Charakterzug der flexiblen Persönlichkeit «, ${ }^{109}$ so wird deutlich, dass selbst die äußerste Konsequenz der dissoziativen Identitätsstörung, nämlich der radikale Verlust der eigenen Biografie und damit Identität, in Anbetracht der von Sennett und Bröckling hervorgehobenen Aspekte des flexiblen Kapitalismus einen Vorteil im Wettbewerb um ökonomische Überlegenheit verspricht. Dieses Versprechen trägt maßgeblich, das galt es aufzuzeigen, zur sukzessiven Entpathologisierung multipler Identitäten bei. ${ }^{110}$ Für die Frage, inwiefern diese Verschiebung im Narrativ sich auch indirekt auf die Diagnose, Erforschung und Behandlung der dissoziativen Identitätsstörung auswirkt, bieten sich weiterführende Studien an.

\section{Literatur}

Aldridge-Morris, Ray 1989. Multiple personality. An exercise in deception. New Jersey: Psychology Press.

Bach, Mischa 2006. Stimmengewirr. Leer: Leda Verlag.

Bauman, Zygmunt 1997. Flaneure, Spieler und Touristen. Essays zu postmodernen Lebensformen. Hamburg: Hamburger Edition.

Besser-Siegmund, Cora; Siegmund, Harry 1991. Coach Yourself. Persönlichkeitskultur für Führungskräfte. Düsseldorf, Wien, New York: Econ.

Bijnsdorp, Liz 1994. Die 147 Personen, die ich bin. Drama und Heilung einer multiplen Persönlichkeit. Stuttgart: Urachhaus.

108 Casey, Wilson 1995 [1991], S. 221.

109 Sennett 1998, S. 79 f.

110 Um präzise zu sein, muss hier zwischen einem psychiatrischen Diskurs im engeren Sinn, der an der klaren Ätiologie, Pathologie und angestrebten Heilung der dissoziativen Identität mittels Fusion festhält, und einem Multiplen-Diskurs im weiteren Sinne unterschieden werden, wie er häufig durch Betroffene, in künstlerischen und philosophischen Auseinandersetzungen sowie in populärkulturellen Verhandlungen des Themas stattfindet. Während ProtagonistInnen des Ersteren es weitgehend zu vermeiden scheinen, vermeintliche Vorteile dissoziativer Identität anzuerkennen, möglicherweise um deren Status als Störung nicht zu hinterfragen, zeigt Letzterer deutliche Tendenzen der Entpathologisierung bis hin zur Affirmierung von Multiplizität.

Leviathan, 42. Jg., 2/2014 
Braude, Stephen E. 1991. First person plural. Multiple personality and the philosophy of mind. London u.a.: Routledge.

Braun, Christina von; Dietze, Gabriele. Hrsg. 1999. Multiple Persönlichkeit. Krankheit, Medium oder Metapher? Frankfurt a. M.: Verlag Neue Kritik.

Bröckling, Ulrich 2007. Das unternehmerische Selbst. Soziologie einer Subjektivierungsform. Frankfurt a. M.: Suhrkamp.

Casey, Joan F.; Wilson, Lynn 1995 [1991]. Ich bin viele. Eine ungewöhnliche Heilungsgeschichte. Reinbek bei Hamburg: Rowohlt Taschenbuch Verlag.

Chase, Truddi 1990. When rabbit howls. New York: Jove Book.

Dale, Peter 1999. "Multiple personality disorder. A sceptical perspective ", in Hidden selves. An exploration of multiple personality, hrsg. v. Walker, Moira; Black, Jennifer A., S. 38-55. Buckingham: Open University Press.

Deleuze, Gilles; Guattari, Félix 1977 [1972]: Anti-Ödipus. Kapitalismus und Schizophrenie I. Frankfurt a. M.: Suhrkamp.

Dennett, Daniel; Humphrey, Nicolas 1989. "Speaking for our selves. An assessment of multiple personality disorder", in Raritan 9, 1, S. 68-98.

Ellenberger, Henri F. 1970. The discovery of the unconscious. The history and evolution of dynamic psychiatry. New York: Basic Books.

Engel, Antke 2009. Bilder von Sexualität und Ökonomie. Queere kulturelle Politiken im Neoliberalismus. Bielefeld: transcript.

Gergen, Kenneth J. 1991. The saturated self. Dilemmas of identity in contemporary life. New York: Basic Books.

Gross, Peter 1994. Die Multioptionsgesellschaft. Frankfurt a. M.: Suhrkamp.

Gunnarsson, Logi 2010. Philosophy of personal identity and multiple personality. New York u.a.: Routledge.

Ha, Kien Nghi 2005. Hype um Hybridität. Kultureller Differenzkonsum und postmoderne Verwertungstechniken im Spätkapitalismus. Bielefeld: transcript.

Hacking, Ian 1995. Rewriting the soul. Multiple personality and the sciences of memory. Princeton: Princeton University Press.

Held, Tilo 1999. »Multiple Persönlichkeitsstörung - ein psychiatriepolitisches Konstrukt? «, in Multiple Persönlichkeit. Krankheit, Medium oder Metapher?, hrsg. v. Braun, Christina von; Dietze, Gabriele, S. 18-31. Frankfurt a. M.: Verlag Neue Kritik.

Herman, Judith L. 1992. Trauma and recovery. The aftermath of violence-from domestic abuse to political terror. New York: Basic Books.

Keupp, Heiner 1999. Identitätskonstruktionen. Das Patchwork der Identitäten in der Spätmoderne. Reinbek bei Hamburg: Rowohlt.

Oxnam, Robert B. 2008 [2005]. Ich bin Robert, Wanda und Bobby: Der Mann mit den elf Persönlichkeiten. Ostfildern: Patmos Verlag.

Phillips, Robert A. 1992 [1987]. »Einleitung «, in Truddi Chase: Aufschrei, S. 7-33. Bergisch Gladbach: Bastei-Lübbe.

Putnam, Frank W. 2013 [1989]. Handbuch Dissoziative Identitätsstörung. Diagnose und psychotherapeutische Behandlung. Lichtenau: G.P. Probst.

Rosenbaum, Milton 1980. »The role of the term schizophrenia in the decline of multiple personality ", in Archives of General Psychiatry 37, S. 1383-1385.

Ross, Colin A. 1989. Multiple personality disorder. Diagnosis, clinical features and treatment. New York: Wiley.

Ruffs, Matt 2004. Set this house in order: a romance of soul. New York, London: Haper Perrenial.

Saß, Henning et al. 1998. Diagnostische Kriterien des Diagnostischen und statistischen Manuals psychischer Störungen DSM-IV. Göttingen u.a.: Hogrefe, Verlag für Psychologie.

Schreiber, Flora Rheta 1996 [1974]. Sybil. Persönlichkeitsspaltung einer Frau. Frankfurt a. M.: Fischer.

Sennett, Richard 1998. Der flexible Mensch. Die Kultur des neuen Kapitalismus. Berlin: Berlin Verlag.

Turkle, Sherry 1995. Life on the screen. Identity in the age of the Internet. New York: Simon \& Schuster. 
Welsch, Wolfgang 1991. »Identität im Übergang. Philosophische Überlegungen zur aktuellen Affinität von Kunst, Psychiatrie und Gesellschaft ", in Ästhetisches Denken, hrsg. v. Welsch, Wolfgang, S. 168-200. Stuttgart: Reclam.

Welsch, Wolfgang 1993. „Subjektsein heute. Zum Zusammenhang von Subjektivität, Pluralität und Transversalität", in Vernunftnähe, Vernunftferne, hrsg. v. Holzhey, Helmut; Leyvraz, Jean Pierre, S. 153-182. Bern: Haupt. 
Zusammenfassung: Der Artikel fragt vor dem Hintergrund der zunehmenden Konstatierung pluraler Identitäten nach den ökonomischen Imperativen, die jenen Erzählungen des Multiplen eingeschrieben sind. Dabei wird der Fokus auf das psychiatrische Narrativ der dissoziativen Identitätsstörung gesetzt, um an diesem exemplarisch eine fortschreitende Ökonomisierung alternativer Subjektivierungsformen zu skizzieren, mit welcher sukzessive eine Umwertung des vormals Devianten hin zum Superioren einhergeht.

Stichworte: dissoziative Identitätsstörung, multiple Persönlichkeit, unternehmerisches Selbst, flexibler Kapitalismus, flexibler Mensch, Identität, Pluralität, ökonomische Imperative, alternative Subjektformen, Devianz - Superiorität

\section{The narrative of the Dissociative Identity Disorder in the context of economic imperatives}

Summary: In view of the fact that there is an increasing confirmation of plural identities, this article explores the economic imperatives inscribed in these narratives of the multiple. It focuses on the psychiatrical diagnosis Dissociative Identity Disorder as an example of the progressive economisation of alternative subjectivities, which resulted in the successive reassessment of what was formerly considered a deviance into something superior.

Keywords: Dissociative Identity Disorder, multiple personality, enterprising self, flexible capitalism, flexible man, identity, plurality, economic imperatives, alternative subjectivities, deviance - superiority

\section{Autorin}

Dr. Rosemarie Brucher

Senior Scientist Theaterwissenschaft

Zentrum für Genderforschung

Universität für Musik und Darstellende Kunst Graz

Brandhofgasse 18

8020 Graz

Österreich

Rosemarie.Brucher@univie.ac.at 